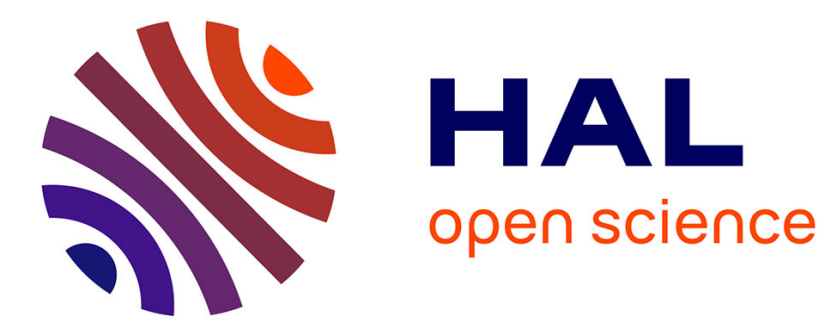

\title{
Full Duplex Prototype of OFDM on GNURadio and USRPs
}

\author{
Wei Zhou, Guillaume Villemaud, Tanguy Risset
}

\section{To cite this version:}

Wei Zhou, Guillaume Villemaud, Tanguy Risset. Full Duplex Prototype of OFDM on GNURadio and USRPs. IEEE Radio Wireless Symposium, Jan 2014, Newport Beach, United States. hal-00920902

\section{HAL Id: hal-00920902 https://hal.inria.fr/hal-00920902}

Submitted on 19 Dec 2013

HAL is a multi-disciplinary open access archive for the deposit and dissemination of scientific research documents, whether they are published or not. The documents may come from teaching and research institutions in France or abroad, or from public or private research centers.
L'archive ouverte pluridisciplinaire HAL, est destinée au dépôt et à la diffusion de documents scientifiques de niveau recherche, publiés ou non, émanant des établissements d'enseignement et de recherche français ou étrangers, des laboratoires publics ou privés. 


\title{
Full Duplex Prototype of OFDM on GNURadio and USRPs
}

\author{
Wei Zhou, Guillaume Villemaud, and Tanguy Risset
}

Université de Lyon, INRIA, INSA-Lyon, CITI-INRIA, F-69621, Villeurbanne, France

\begin{abstract}
Full-duplex is a technology in telecommunication domain that can perform transmitting and receiving at the same time and in the same frequency band. The major obstacle of full-duplex is the self-interference (SI). Some previous works have focused on the SI cancellation in radio frequency ( $R F)$, and generally on a narrowband signal model. We have implemented a full-duplex prototype with orthogonal frequency-division multiplexing (OFDM) technology on GNURadio and Universal Software Radio Peripherals (USRP). We focus on the baseband, namely the digital part of the SI cancellation. Our testbed can achieve a digital cancellation of $27 \mathrm{~dB}$. After the cancellation, the signal of interest can achieve a bit error rate (BER) in the scale of $10^{-5}$ at 4 meters, which is very close to the performance of half-duplex. This is, to our knowledge, the first full duplex implementation for OFDM technology.
\end{abstract}

\section{INTRODUCTION}

In telecommunication systems, normally the usage of the radio channel resource should be doubled to realize bidirectional communication. This kind of technology is called half-duplex. The full-duplex, on the other hand, permits the terminal to receive and transmit at the same time, in the same band of frequency. That makes the mechanism of access to media and spectrum allocation much easier and more flexible. It is also helpful for cognitive radio and wireless sensor networks.

A typical full-duplex transceiver requires at least one transmitting (TX) antenna and one receiving (RX) antenna. Normally the distance between the two antennas of the same node is much shorter than the distance between the two nodes. Thus the self-interference (SI) is significantly more important than the signal of interest. According to the calculation made in [1], this difference is $73 \mathrm{~dB}$, which makes SI cancellation the key issue in realizing a fullduplex system.

As SI cancellation is the most critical problem in the research of full-duplex, many researchers are involved in this topic. However, most of the works are focused on narrowband signal. We tend to analyze the performance of full-duplex on orthogonal frequency-division multiplexing (OFDM), which is a widely used standard in nowadays wireless telecommunication, e.g. in LTE and 802.11. We have chosen GNURadio and the Universal Software Radio Peripherals (USRP) as our software and hardware platform respectively. This combination is a typical Software Defined Radio (SDR) solution.

\section{StATE OF THE ART}

Some related works [2][3] tend to divide the SI cancellation into three major categories: antenna cancellation, RF cancellation and digital cancellation.

In [2], antenna cancellation was achieved by placing two TX antennas asymmetrically at $\mathrm{d}$ and $\mathrm{d}+\lambda / 2$ distance from the RX antenna. This allows the transmit signals to add $\pi$ out of phase and hence cancel each other. Analog cancellation is also proposed, which requires knowledge of the SI channel to create a copy of the SI signal in the RF domain and cancel it before the signal is digitized. Digital cancellation utilizes the digital samples of the transmitted signal and subtracts them from the received samples, removing up to $25 \mathrm{~dB}$ of SI in their work.

In [3], the authors propose a novel mechanism, balun cancellation, which uses signal inversion, through a balun (balanced/unbalanced) circuit. Being a method of analog cancellation, it profits from the knowledge of the transmission to cancel SI in the RF signal, before it is digitized.

Melissa Duarte and Ashutosh Sabharwal discussed the existing approaches and proposed a experimental result on WARPLab framework in their article [4]. Melissa Duarte also proposed in her thesis [5] a detailed plan of implementing a full-duplex system for 802.11 transceiver. But in these works, their effort is always focused on narrowband.

\section{PlatfForm}

We selected GNURadio and USRP as our platform of software and hardware respectively.

GNU Radio is a free and open-source software development toolkit that provides signal processing blocks to implement software radios [6].

The USRP is a family of products designed and supported by Ettus Research, a National Instrument (NI) company. USRP products are used by engineers worldwide and remain the top choice in software defined radio hardware [7]. We have chosen NI USRP 2922 as our testbed components. The daughter board of the NI USRP 2922 supports a frequency band of $400 \mathrm{MHz}$ to $4.4 \mathrm{GHz}$ [8]. To avoid potential collision with the existing 802.11 or bluetooth signal in the widely used ISM band of $2.4-2.5 \mathrm{GHz}$, we have chosen $2.15 \mathrm{GHz}$ as our testing frequency.

It should be noted that the GNURadio is not the only solution for implementing an SDR platform with USRP. The NI Company also provided LabVIEW as a very 
powerful SDR developing toolkit for USRP products. In fact, the EPFL of Switzerland has already implemented their full-duplex prototype on the USRP and LabVIEW [9]. They had achieved a cancellation about $30 \mathrm{~dB}$ in the same frequency band as we used. But their experiment still focused on a narrowband signal, not wideband such as OFDM.

In our SI cancellation test, we have set up a full-duplex transceiver with two USRPs, connected by a MIMO cable. One USRP served as the transmit path, the other act as the receive path. In the part of full-duplex test, a third USRP is needed to act as the far-end transmitter that diffuses the signal of interest. Fig. 1 presents the scheme of the testbed.

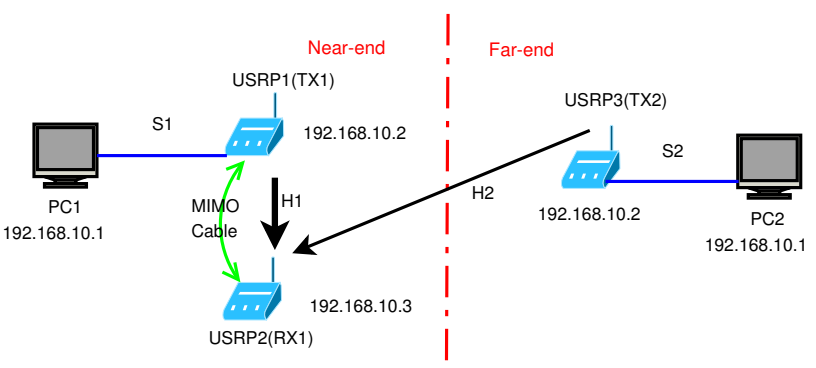

Fig. 1. Full-Duplex testbed scheme

\section{DESIGN}

Figure 2 represents the proposed flow chart of fullduplex solution with OFDM. Both the conventional transmit path and the receive path from GNURadio take part in this procedure. The transmit path gives us the result of adding preamble of the being transmitted data, and it should be rebuilt according to the characteristics of the channel. The receive path is in charge of detecting the received signal, making frequency offset compensating and FFT demodulation. Inside the new formed ofdm_frame_rebuilder function, sub-carrier alignment and channel estimation are taking place and rebuild the being transmitted data obtained from the transmit path according to the characteristics of the channel. Then it will pass through the IFFT to be transformed to time domain, and take a delay factor that is also estimated by ofdm_sync function of the receive path to make sure that the two signals are well aligned in time domain. At last the rebuilt signal will be subtracted from the original received signal. Then the rest part after the subtraction, which is considered to be the data of interest, will be passed to the conventional receive path to be demodulated.

\section{EXPERIMENTS AND ANALYSES}

The experiment is divided into two parts. Firstly we focus on the performance of SI cancellation. Without the participation of the third USRP, we only test the cancellation performance of the near-end, which is composed of two USRPs and a MIMO cable. In the second part of the

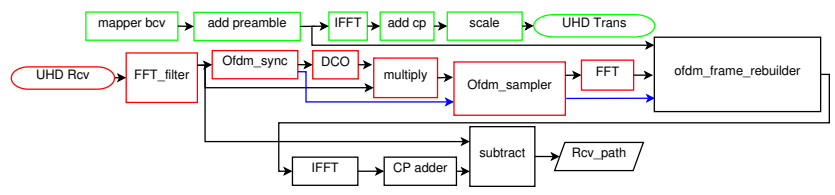

Fig. 2. Flow chart of ofdm_full_duplex

test, we add the third USRP into our test to act as the far-end.

\section{A. SI Cancellation Experiment}

In this part of test, our major measure metric is the cancellation rate, which is defined in making use of error vector magnitude (EVM) as following:

$$
E V M=\frac{\|e\|}{\|V\|} \approx \frac{\|e\|}{\|V r\|}
$$

where $V$ represents the vector that we rebuild from the information provided by the transmitting path, $V r$ represents the vector that we actually received, thus $e$ means the error between them, i.e. the residual SI. Therefore the average rate of cancellation is:

$$
\hat{C}=\frac{1}{n m} \sum_{i=1}^{n} \sum_{j=1}^{m} \frac{1}{\left(E V M_{i j}\right)^{2}}
$$

where $\mathrm{m}$ represents the number of sub-carriers in one OFDM symbol, and $\mathrm{n}$ represents the total number of OFDM symbols during our test. In this way we have got the cancellation rate of our testbed that reaches $27 \mathrm{~dB}$.

\section{B. Full-Duplex Experiment}

In this part of the experiment, we make use of three USRPs. Both the near-end and the far-end transmit their own signals simultaneously. The major metric is bit error rate (BER) of the signal of interest after canceling the SI.

As shown in Fig. 3, we can observe that the BER declines as the TX Gain factor raises. As the distance get farther, the performance is getting worse.

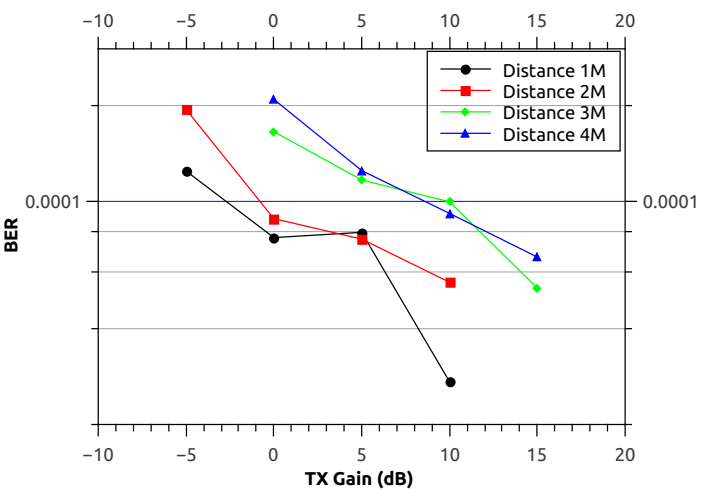

Fig. 3. BER with different TX Gain and distance settings 
Note that the TX Gain means the difference between TX Gain at the far-end and the TX Gain at the near-end, namely the relative TX Gain. We found that our full-duplex experiment can tolerate the distance less than 4 meters. We tend to try some longer distances for example 5 meters, but the preamble can hardly be sensed at that distance with the presence of SI.

Furthermore we tend to analyze the result in making use of SINR, which takes both the path loss caused by the distance and the TX gain into account. Note that the interference is much more powerful than the additive noise, so we tend to suppose that the noise level is negligible. Thus the SINR after the cancellation is supposed to be:

$$
S I N R_{\text {residual }} \approx S I R_{\text {residual }}=P_{\text {signal }}-\left(P_{\text {interference }}-\hat{C}\right)
$$

In this sense, we can rebuild the above figure in another manner that compares the BER with $\mathrm{SIR}_{\text {residual }}$.

As shown in Fig. 4, the different lines show the same trend: as the relative SINR increases, the BER declines. The lines cross with each other, which mean the relative SINR has well unified the pass loss and TX gain factors.

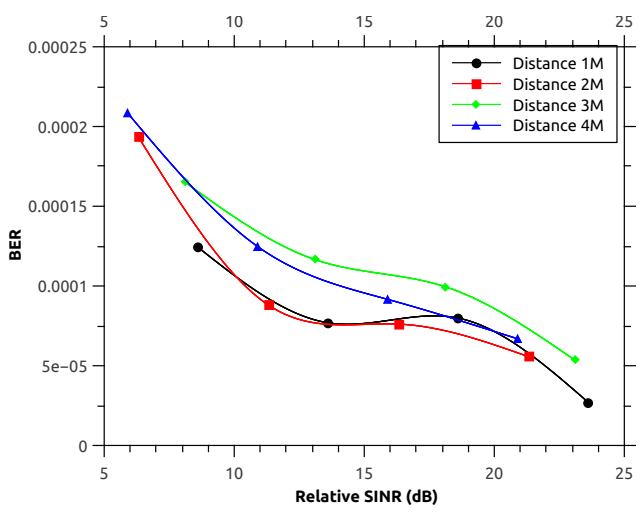

Fig. 4. BER with $\operatorname{SIR}_{\text {residual }}$

As shown in Fig. 5, we compared the BER performance for several modulation settings provided by the GNURadio. As we raise the level of modulation, i.e. from BPSK to 8PSK, the BER raises. For the higher modulation levels, the difference among the constellation points decreases, thus the possibility of misjudging must be increasing. As a comparison, we also append the performance of halfduplex for each modulation as a reference. We can observe that our full-duplex result is worse than that of half-duplex, which means at the same SINR level, the residual SI is more harmful than additive noise. In addition, we attached the theoretical BER for BPSK modulation in OFDM with Rayleigh multipath channel [10] and AWGN channel [11] as a reference. As we can observe from Fig. 5, both the results of half-duplex and full-duplex are between the theoretical performance of the two channel models.

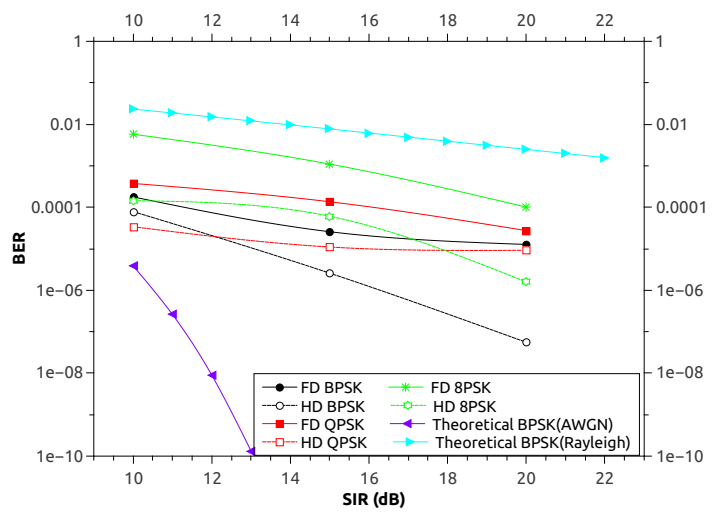

Fig. 5. BER with different modulation settings for full duplex (FD) and half duplex (HD).

\section{Conclusion}

We have achieved a prototype of full-duplex transceiver, which is by now the first OFDM version full-duplex transceiver on GNURadio and USRP platform. Some related works already exist in implementing the full-duplex on narrowband channel models, but our contribution is applying the full-duplex idea on a more practical wideband signal model. We focused on the digital cancellation, which reached a cancellation of $27 \mathrm{~dB}$. We also measured the BER of the signal transmitted from the far-end transmitter as a source of interest after the SI cancellation. The best result is in the scale of $10^{-5}$, which is very close to the performance of half-duplex.

\section{REFERENCES}

[1] E. A. M. A. Khojastepour, K. Sundaresan, S. Rangarajan, and M. Chiang, "Midu: Enabling mimo full duplex," Mobicom'12, no. 257-268, 2012.

[2] J. Choi, M. Jain, K. Srinivasan, P. Levis, and S. Katti, "Achieving single channel, full duplex wireless communication," MobiCom, September 2010.

[3] M. Jain, J. I. Choi, T. M. Kim, D. Bharadia, and S. Seth, "Practical, real-time, full duplex wireless," MobiCom, September 2011.

[4] M. Duarte and A. Sabharwal, "Full-duplex wireless communications using off-the-shelf radios: Feasibility and first results," Conference Record of the Forty Fourth Asilomar Conference, no. 1558 - 1562, 2010.

[5] M. Duarte. Full-duplex wireless: Design, implementation and characterization. [Online]. Available: http://warp.rice.edu/trac/ raw-attachment/wiki/DuartePhDThesis/MDThesis.pdf

[6] The definition of gnuradio. [Online]. Available: http://gnuradio. org/redmine/projects/gnuradio/wiki

[7] E. Research. The definition of usrp. [Online]. Available: http://www.ettus.com/

[8] Specification of ni usrp 2922. [Online]. Available: http://sine.ni. com/nips/cds/print/p/lang/fr/nid/211243

[9] B. Gozcu, "Full duplex wireless radio: Investigation and implementation using universal software radio peripherals," obtained by academic cooperation.

[10] Ber for bpsk in ofdm with rayleigh multipath channel. [Online]. Available: http://www.dsplog.com/2008/08/26/ ofdm-rayleigh-channel-ber-bpsk/

[11] Bpsk ber with ofdm modulation. [Online]. Available: http: //www.dsplog.com/2008/06/10/ofdm-bpsk-bit-error/ 